Received: 13.08 .2020

Revised: 11.09 .2020

Accepted: 09.10.2020

DOI: $10.17804 / 2410-9908.2020 .5 .006-016$

\title{
A PRECISION LOW-FREQUENCY ANALOG-TO-DIGITAL CONVERTER FOR ADVANCED SYSTEMS OF TECHNICAL DIAGNOSTICS AND NONDESTRUCTIVE TESTING
}

\author{
N. A. Lukin ${ }^{\text {a) }}$ and L. S. Rubin ${ }^{\text {b* }}$ \\ Institute of Engineering Science, Ural Branch of the Russian Academy of Sciences, \\ 34, Komsomolskaya St., Ekaterinburg, 620049, Russian Federation \\ a) (iD http://orcid.org/0000-0001-6326-217X; \\ b) $\triangle$ rubin281@yandex.ru \\ *Corresponding author. E-mail: rubin281@yandex.ru \\ Address for correspondence: 34 Komsomolskaya St., Ekaterinburg, 620049, Russian Federation \\ Tel.: +7 (919) 3871531
}

The main results of the development of a precision low-current meter based on a nonlinear ADC are presented. It is shown that the application of the method of nonlinear analog-to-digital conversion and a controlled voltage level at the non-inverting input of an integrating operational amplifier allows both high sensitivity and a wide dynamic range with a small spread of output codes. The use of the laboratory sample of the meter for cyclic tensile testing of steel specimens is described.

Keywords: nonlinear analog-to-digital converter, nanoampere current meter, integrating operational amplifier, magnetostriction measurement.

\section{References}

1. Aaltonen L. Integrated Interface Electronics for Capacitive MEMS Inertial Sensors, Doct. Diss., Aalto Univ., Sc. Sci. Tech., Fac. Electr., Comm. Autom., Dep. Micro Nanosci., 2010, 152 p.

2. $\quad$ Boyden E., Rifai O.El., Hubert B., Karpman M., Roberts D. A High-Performance Tunneling Accelerometer. MIT Term Project Paper 6.777, Introduction to Microelectromechanical Systems, Spring, 1999, 58p.

3. Osaki Yuji, Hirose Tetsuya, Kuroki Nobutaka, and Numa Masahiro. TemperatureCompensated Nano-Ampere Current Reference Circuit with Subthreshold Metal-OxideSemiconductor Field-Effect Transistor Resistor Ladder. Jpn. J. Appl. Phys., vol. 50, no. 4S, pp. 04DE08-1-6. DOI: 10.1143/JJAP.50.04DE08.

4. Linares-Barranco B., Serrano-Gotarredona T., Serrano-Gotarredona R., SerranoGotarredona C. Current Mode Techniques for Sub-pico-Ampere Circuit Design. Analog Integrated Circuits and Signal Processing, 2004, 38, pp. 103-119.

1. Digital processors limited by power; what's the upside? Available at: https://phys.org/news/2012-08-digital-processors-limited-power-upside.html

5. Gelman M.M. Sistemnye analogo-tsyfrovye preobrazovateli i protsessory signalov [System Analog-to-Digital Converters and Signal Processors]. Moscow, Mir Publ., 1999, 559 p. (In Russian).

6. Lukin N.A., Rubin L.S. Using nonlinear functional analog-to-digital conversion for precision measurements of small electrical quantities in real time. Giroskopiya i Navigatsiya, 2014, no. 4 (87), pp. 131-141. (In Russian). 
7. Integrating analog-to-digital converter for measuring small electrical signals. Patent RF 2693647 C1, 2019. (In Russian).

8. Gorkunov E.S., Povolotskaya A.M., Zadvorkin S.M., Putilova E.A., Mushnikov A.N., Bazulin E.G., Vopilkin A.K. Some features in the behavior of magnetic and acoustic characteristics of hot-rolled 08G2B steel under cyclic loading. Russian Journal of Nondestructive Testing, 2019, vol. 55, no. 11, pp. 827-836. 
Подана в журнал: 13.08 .2020

УДК 681.325,621.317.311

DOI: $10.17804 / 2410-9908.2020 .5 .006-016$

\title{
ПРЕЦИЗИОННЫЙ НИЗКОЧАСТОТНЫЙ ПРЕОБРАЗОВАТЕЛЬ «АНАЛОГ-КОД» ДЛЯ ПЕРСПЕКТИВНЫХ СИСТЕМ ТЕХНИЧЕСКОЙ ДИАГНОСТИКИ И НЕРАЗРУШАЮЩЕГО КОНТРОЛЯ
}

\author{
Н. А. Лукин ${ }^{\text {a) }}$ Л. С. Рубин ${ }^{\text {б)* }}$ \\ Институт машиноведения УрО РАН, \\ 34, ул. Комсомольская, г. Екатеринбург, 620049, Российская Федерация \\ a) (iD http://orcid.org/0000-0001-6326-217X; \\ b) rubin281@yandex.ru \\ *Ответственный автор. Электронная почта: rubin281@yandex.ru \\ Адрес для переписки: ул. Комсомольская, 34, г. Екатеринбург, Российская Федерация \\ Тел.: 8-919-387-15-31
}

Приводятся основные результаты разработки прецизионного измерителя малых токов на базе нелинейного аналого-цифрового преобразования (АЦП). Применение разработанного метода и управляемого уровня напряжения на неинвертирующем входе интегрирующего операционного усилителя позволяет одновременно достичь высокой чувствительности и широкого динамического диапазона при малом разбросе выходных кодов. Описано применение лабораторного образца измерителя при циклических испытаниях стальных образцов на растяжение.

Ключевые слова: нелинейный аналого-цифровой преобразователь, измеритель наноамперного тока, интегрирующий операционный усилитель, измерение магнитострикции.

\section{1. Введение}

Развитие методов и средств неразрушающего контроля в настоящее время связано с прогнозированием остаточного ресурса контролируемых объектов, что требует реализации систем технического диагностирования. Современные средства технической диагностики включают в свой состав устройства цифровой обработки сигналов, что требует преобразования выходных сигналов сенсоров в цифровой код. К настоящему времени разработана большая номенклатура разнообразных аналого-цифровых преобразователей, реализованных в виде микросхем.

Можно выделить следующие области применения, в которых в настоящее время сформулированы наиболее жесткие требования к АЦП:

- встроенная электроника микромеханических акселерометров $[1,2]$;

- системы контроля параметров сверхбольших интегральных схем в процессе их изготовления и эксплуатации [3];

- прецизионные сенсоры для регистрации физических и химических процессов с очень малыми значениями энергии [4].

Кроме того, развитие измерительных систем реального времени выявляет необходимость прецизионного измерения малых электрических параметров. Краткий анализ состояния разработок систем измерения малых параметров в реальном времени [7] показывает, что необходимо обеспечивать выполнение взаимно противоречивых требований:

- необходимо добиваться измерения малых и сверхмалых токов (порядка нанои пикоампер) с относительной суммарной погрешностью порядка долей, \%; 
- требуется обеспечивать измерения в динамическом диапазоне до 100 дБ;

- процесс измерения должен производиться в режиме жесткого реального времени;

- необходимо обеспечить измерения одновременно от нескольких разных сенсоров.

Одновременное удовлетворение всем перечисленным требованиям с использованием существующих подходов, основанных на усилении входного сигнала и последующем аналогоцифровом (АЦ)-преобразовании, практически невозможно. Поэтому представляется целесообразным использование функииональных возможностей микроэлектроники, которые могут реализовать на аналоговом уровне первичную обработку входного сигнала с тем, чтобы как минимум существенно упростить АЦ-преобразование. Это направление на современном и будущем этапах развития микроэлектроники в определенной степени возвращает достаточно старые концепции АЦП, которые успешно развивались еще в 50-60-х гг. XX в. во многих научных школах и коллективах разработчиков нашей страны. К таким идеям все чаще приходят исследователи и разработчики современных систем реального времени, когда с помощью чисто цифровых подходов уже не удается добиться либо высокой точности, либо широкого диапазона изменения измеряемых параметров. В настоящее время в ряде случаев предпринимаются попытки реализовать задачи с помощью аналоговых процессоров. В частности, агентство DARPA начало финансирование разработки экспериментального образца системы наблюдения и разведки на основе оптических сенсоров [5]. В этом проекте прорабатывается вариант использования аналоговых решающих модулей для реализации алгоритмов, которые еще недавно считались чисто «цифровыми» в плане применения элементной базы.

В настоящей статье описаны результаты разработки многоканальной прецизионной измерительной системы сбора данных (ССД), представленных в форме входных токов малой величины, для систем реального времени. АЦП реализуется на основе широко применяемой операции интегрирования. При этом внимание уделяется использованию функциональных возможностей аналоговой электроники с целью уменьшения погрешности измерения и увеличения диапазона измеряемых величин. Операция интегрирования выполняется в течение заданного интервала времени, что ограничивает быстродействие АЦП интегрирующего типа.

\section{2. Нелинейная шкала измерений}

Измерение малых токов в ответственных применениях всегда связано с необходимостью уменьшить влияние неизбежно присутствующих помех аддитивного характера. С целью снижения влияния этих помех был выбран один из наиболее точных - метод АЦП с двухтактным интегрированием [6]. Суть метода состоит в том, что в первом такте измеряемый входной ток интегрируется на емкости операционного усилителя-интегратора и за определенный заранее отрезок времени преобразуется в уровень напряжения на емкости. Во втором такте производится разряд этой емкости до некоторой заранее заданной величины напряжения на обкладках. При этом изменение напряжения на выходе операционного усилителя-интегратора во втором такте преобразования определяется выбором способа разряда интегрирующей емкости. Высокая точность метода определяется тем, что одна и та же емкость участвует в процессах заряда-разряда, при этом одновременно производится усреднение аддитивной смеси входного тока и помех.

В измерителях с двухтактным интегрированием чаще всего применяются линейные измерительные шкалы. В этом случае для обеспечения большого динамического диапазона измерения, например 100 дВ, необходимо существенно увеличивать напряжение источников питания, что для аппаратуры с автономным питанием нежелательно. Увеличения динамического диапазона измеряемой величины добиваются путем применения нелинейной шкалы, которая реализуется с помощью кусочно-линейной аппроксимации, при которой линейность шкалы с некоторой постоянной крутизной сохраняется на определенном интервале измеряемых величин, а при выходе за этот интервал крутизна шкалы меняется, сохраняя постоянное значение до достижения следующей границы интервала измеряемой величины. Известным 
недостатком кусочно-линейной аппроксимации является значительная погрешность измерений в местах переключения интервальных шкал. Это обусловлено наличием так называемой «мертвой» зоны в компараторах, которая увеличивает суммарную погрешность измерений и более существенно зависит от величины внешнего шума, чем погрешность для любой другой точки линейных сегментов приближения нелинейной шкалы. Этого недостатка принципиально лишены гладкие нелинейные шкалы, которые являются одним из предметов рассмотрения в нашей работе.

Для формирования измерительной шкалы используется источник тока разряда емкости интегратора, у которого ток меняется во времени. Вид нелинейности шкалы определяется заранее комбинацией параметров (номиналов) элементов цепи разряда емкости интегратора и изменения тока разряда. Основой функционирования измерителя является нелинейное АЦ-преобразование входных токов в цифровые коды, при этом в качестве функциональной шкалы преобразования в каждом канале измерения выбрана параболическая шкала вида $N=k\left(I_{i n}\right)^{m}$, где $k-$ масштабный коэффициент, $I_{i n}-$ входной ток [A]; $m<1$. В [7] показано, что при любых значениях измеряемого тока выбранная шкала обеспечивает меньшую относительную суммарную погрешность измерения по сравнению с линейным вариантом $(m=1)$, широко используемым в настоящее время.

Применение АЦП интегрирующего типа позволяет не только эффективно снизить влияние аддитивных случайных шумов на разброс выходных кодов, но и заменить цифровое интегрирование входного сигнала при необходимости его дальнейшей обработки. В этом случае удается избежать погрешностей численных методов интегрирования.

Важное значение имеет борьба с влиянием электромагнитного фона, который практически всегда присутствует при технической диагностике исследуемых объектов в реальных условиях их эксплуатации. Электромагнитный фон влияет на входные цепи измерителей малых электрических сигналов, действуя как аддитивная помеха. Для снижения этого влияния дополнительно к двухтактному интегрированию применен компенсационный метод. Суть компенсационного метода состоит в том, что создается электрическая цепь, симметричная входной цепи измерительного устройства, а на ее выходе формируется электрический сигнал, вызванный только действием электромагнитного фона, и этот сигнал затем вычитается из смеси сигнала и помехи на входе измерительных цепей преобразователя «ток-код». Структурная схема АЦП интегрирующего типа с параболической функциональной шкалой, реализующая компенсационный метод подавления влияния электромагнитного фона, приведена в [8]. Эффективность компенсационного метода целиком определяется примененной электрической схемой входных цепей измерителя.

\section{3. Макет нелинейного преобразователя «аналог-код»}

С целью подтверждения эффективности предложенного способа преобразования на основе результатов работ по компьютерному моделированию алгоритмических и схемотехнических решений был создан макет 4-канального преобразователя «наноамперный ток-код» с нелинейной шкалой преобразования. На рис. 1 приведена структурная схема 4-канального преобразователя «наноамперный ток-код».

Структурная схема состоит из аналоговой и цифровой частей. Аналоговая часть содержит четыре измерительные ячейки и общий для всех ячеек генератор пилообразного напряжения. На выходе каждого канала формируется короткий импульс, временное положение которого определяется интегралом от измеряемого электрического тока. Цифровая часть разработана на основе стандартной платы STM32L-Discovery. B цифровой части формируются все служебные сигналы, необходимые для организации двухтактного интегрирующего преобразования входного тока во временное положение импульса, а также преобразования временного положения импульса в цифровой код, который через USB-разъем поступает в память подключаемого через этот разъем компьютера. 


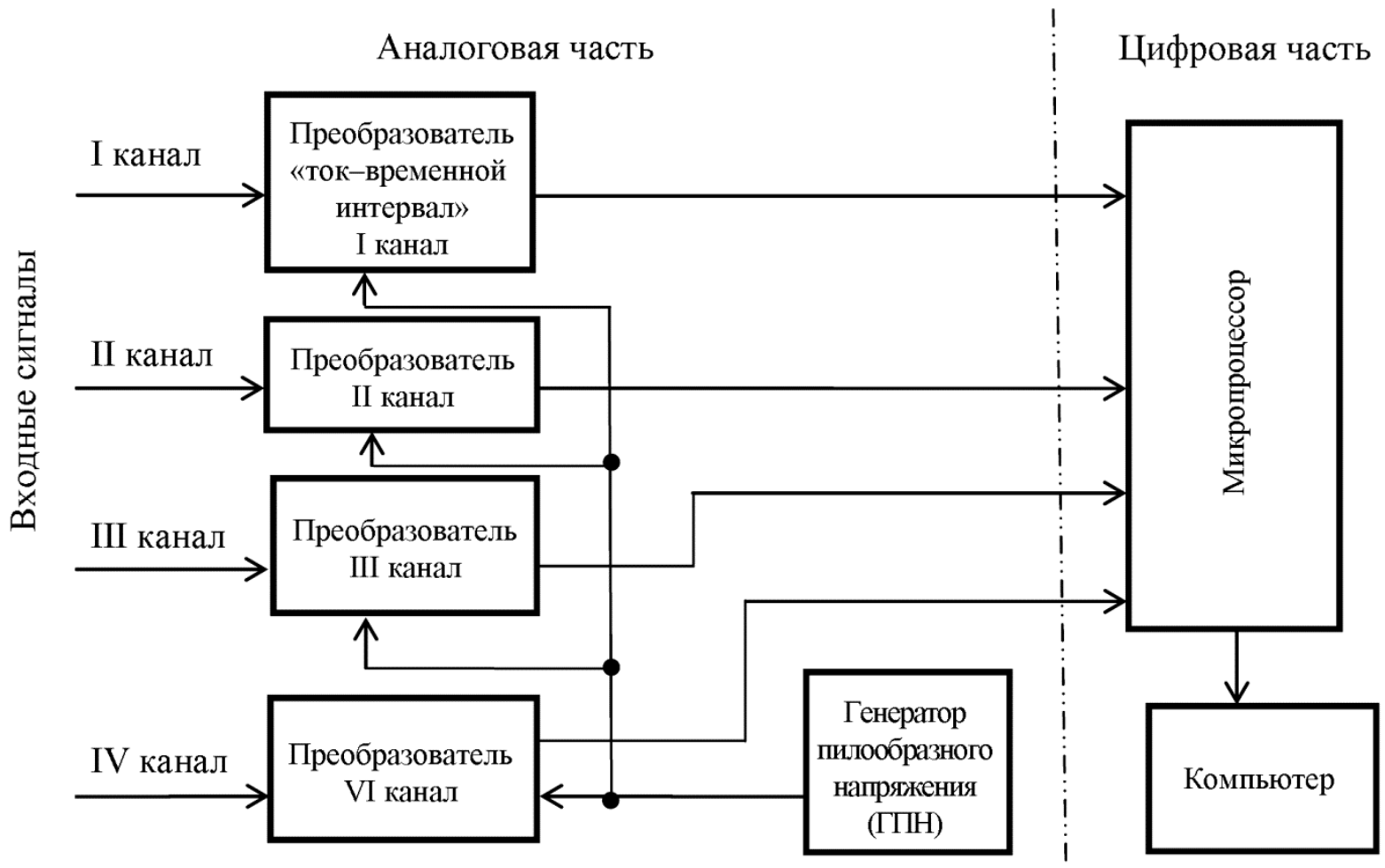

Рис. 1. Структурная схема 4-канального преобразователя «наноамперный ток-код»

На рис. 2 представлена функциональная схема одного канала преобразователя «аналог-временной интервал», на рис. 3 приведены временные диаграммы, поясняющие ее функционирование. Работа схемы осуществляется циклами длительностью 10 мс. Каждый цикл состоит из двух тактов: первый такт длится 3,5 мс, второй - 6,5 мс (рис. $3 a$ ). Соответствующие управляющие импульсы от микропроцессора поступают на контакт ЗАПУСК. Входной измеряемый ток через контакт ВХОДНОЙ ТОК и цепь заряда емкости заряжает емкость $C$ в течение всего первого такта. Операционный усилитель $(\mathrm{OУ)} \mathrm{и} \mathrm{включенная} \mathrm{парал-}$ лельно ему емкость $C$ являются интегратором, поэтому выходное напряжение ОУ линейно изменяется во времени (рис. 3 б), а на емкости $C$ накапливается электрический заряд.

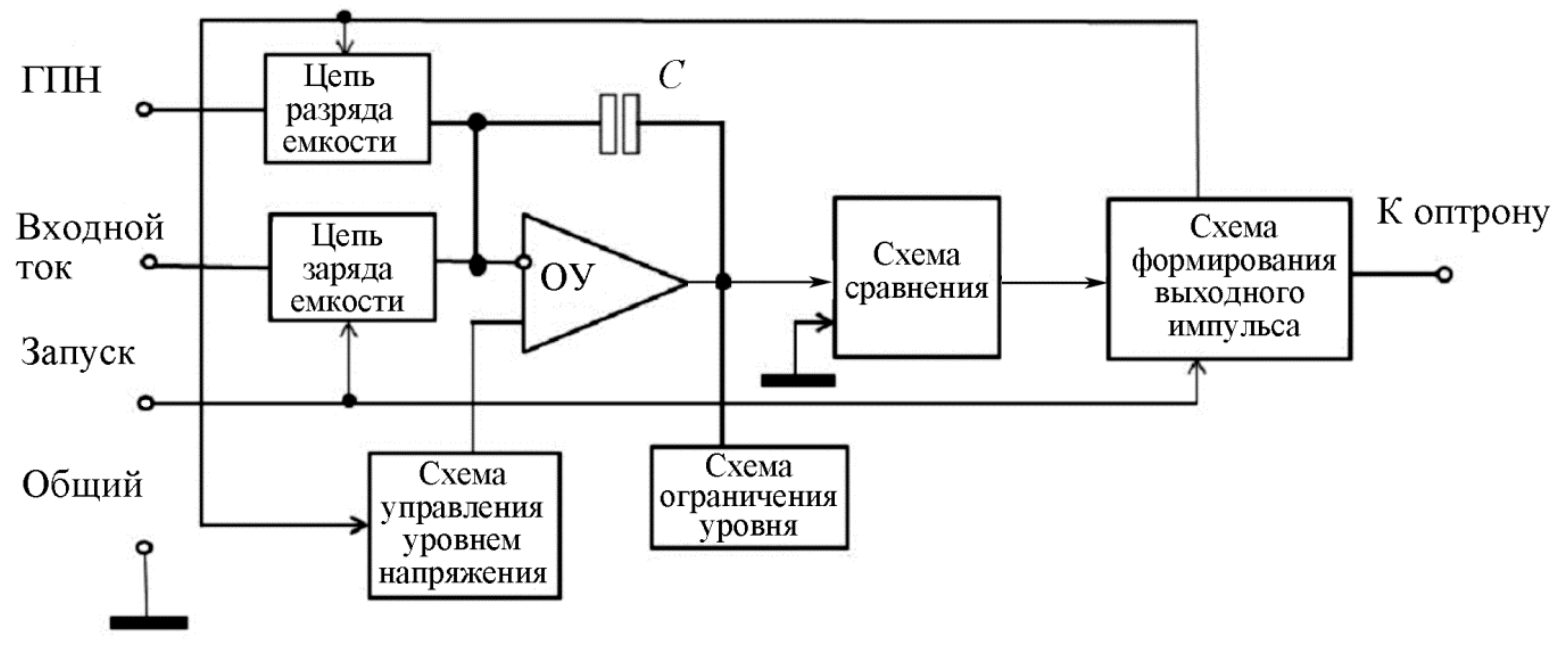

Рис. 2. Функциональная схема одного канала преобразователя «аналог-временное положение импульса» аналоговой части макета 


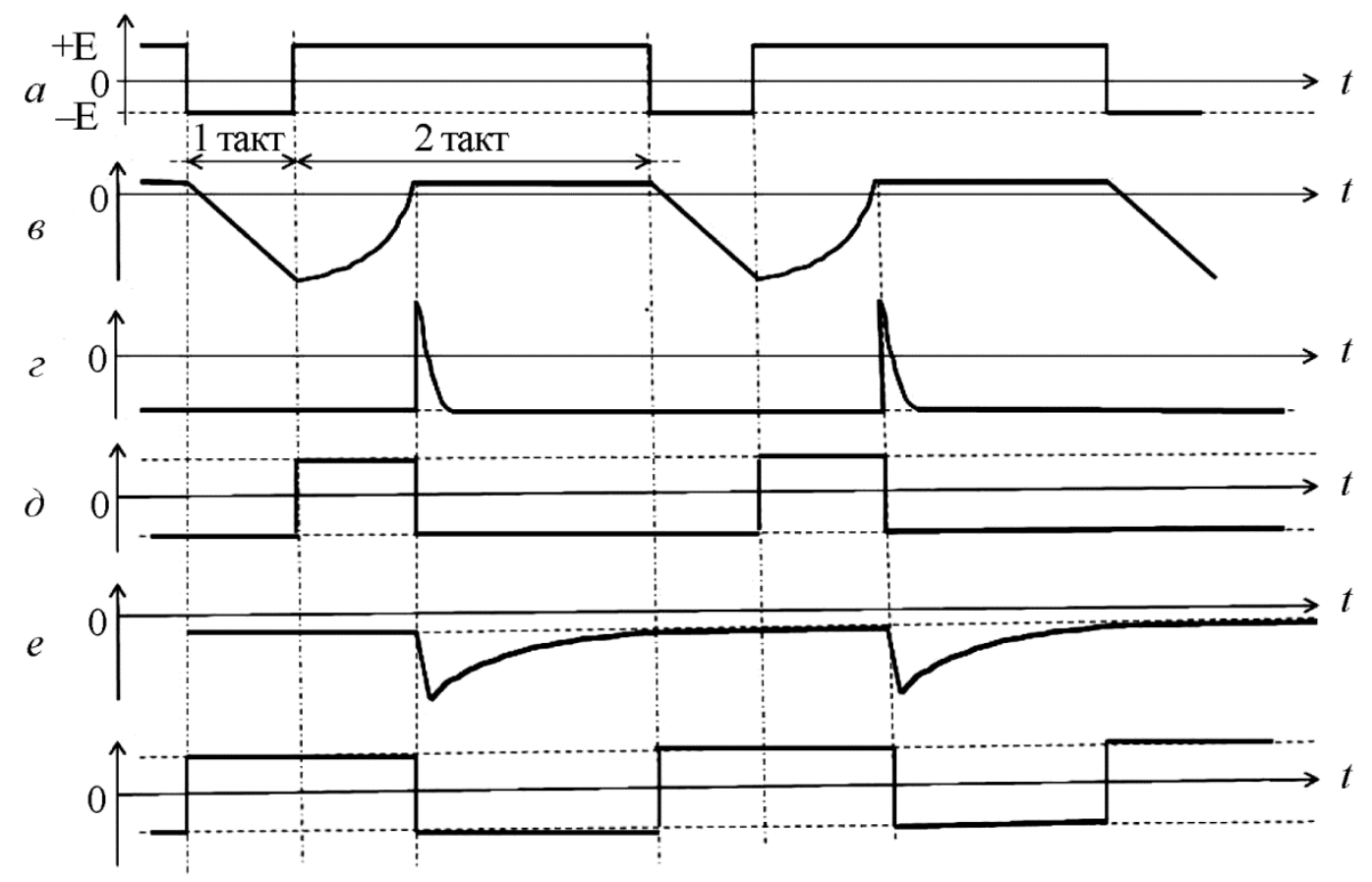

Рис. 3. Временные диаграммы напряжений в разных точках функциональной схемы рис. 2

По окончании управляющего импульса ЗАПУСК начинается второй такт преобразования, цепь заряда емкости прерывает поступление входного тока на заряд емкости $C$, которая начинает разряжаться через цепь разряда. Величина тока разряда емкости $C$ определяется напряжением, формируемым на контакте ГПН, соединенным через резистор с выходом генератора пилообразного напряжения (рис. 1). Это и обеспечивает нелинейность разряда емкости, при этом $m=0,5$.

К выходу ОУ подключены схема сравнения и схема ограничения уровня. Схема сравнения находится в режиме ожидания до тех пор, пока процесс разряда емкости $C$ не приведет к тому, что уровень напряжения на выходе ОУ становится сравнимым с постоянным уровнем, который подается в схеме макета на контакт ОБЩИЙ. Момент сравнения зависит от уровня напряжения, до которого зарядилась емкость интегратора к концу первого такта преобразования, и от величины скорости изменения тока разряда.

Этот момент фиксируется схемой сравнения, которая формирует на выходе импульс положительной полярности (рис. 3 в). Этим импульсом запускается схема формирования выходного сигнала, при этом:

1) формируется выходной импульс канального преобразователя «ток - временной интервал», электрически согласованный с входными цепями микропроцессора цифровой части макета, и поступающий на выходной контакт К ОПТРОНУ;

2) формируется импульсный сигнал, управляющий подключением генератора пилообразного напряжения через цепь разряда емкости к емкости $C$ (рис. 3 2), передний фронт которого совпадает с окончанием первого такта преобразования, а задний фронт во времени совпадает с выходным импульсом схемы сравнения (рис. 3 в). Этот же импульсный сигнал используется для управления схемой управления уровнем напряжения;

3) синхронизируется схема формирования выходного сигнала импульсом ЗАПУСК.

Работу схемы ограничения уровня и схемы управления уровнем напряжения рассмотрим совместно. Схема управления уровнем напряжения подстраивает уровень напряжения на неинвертирующем входе ОУ. Стандартные схемы включения ОУ в режиме интегратора предполагают постоянство напряжения на неинвертирующем входе. Обычно это нулевой уровень относительно напряжений источников питания, в функциональной схеме он уста- 
новлен на контакте ОБЩИЙ. Однако такое включение не позволяет использовать свойство ОУ подавлять синфазные помехи, если они одновременно присутствуют на инвертирующем и неинвертирующем входах ОУ. Для обеспечения такой возможности необходимо изменить схему подключения неинвертирующего входа, что и осуществляется схемой управления уровнем напряжения.

В основе действия схемы управления лежит подключение к неинвертирующему входу запоминающей емкости, на которой формируется и запоминается электрический потенциал. Поскольку в ОУ всегда обеспечивается линейный режим работы, то потенциал, устанавливающийся на неинвертирующем входе, с высокой точностью повторяется на инвертирующем входе. При этом выходной импульс канального преобразователя формируется в момент времени, который наступает после начала второго такта преобразования даже в том случае, если входной ток равен нулю. Это обеспечивает величину нижней границы динамического диапазона измерения, которая в цифровой форме на цифровом выходе преобразователя отображается ненулевым кодом.

Сигнал на неинвертирующем входе ОУ показан на рис. 3 д. В схеме управления уровнем напряжения формируется небольшой отрицательный уровень на интервале времени от начала первого такта до момента сравнения (рис. 3 e). В течение этого отрезка времени на запоминающей емкости устанавливается небольшой неизменный отрицательный потенциал, который повторяется на инвертирующем входе, что добавляет к входному току некоторый постоянный ток, который и обеспечивает ненулевой код при нулевом входном токе.

Схема сравнения формирует импульс в момент превышения сигналом на выходе ОУ нулевого уровня, который затем фиксируется схемой ограничения уровня до момента начала первого такта следующего цикла преобразования. Фиксация уровня на выходе ОУ позволяет стабилизировать остаточный заряд емкости $C$ до начала первого такта любого цикла преобразования, а также обеспечивает линейный режим работы ОУ во втором такте преобразования от момента сравнения до начала первого такта преобразования следующего цикла.

От момента формирования импульса сравнения до начала первого такта следующего цикла преобразования в схеме управления уровнем напряжения разблокируются цепи «заряда-разряда» запоминающей емкости. В результате напряжение на неинвертирующем входе изменяется, немного снижаясь в отрицательную область в начале процесса перезаряда запоминающей емкости, а затем плавно изменяясь до уровня, который был в начале первого такта преобразования. К началу первого такта второго цикла преобразования потенциал на неинвертирующем входе восстанавливается. Поскольку режим работы ОУ линеен, то это обеспечивает постоянство и практическое равенство уровней на неинвертирующем и инвертирующем входах ОУ в течение всего времени «заряда-разряда» емкости в любом цикле преобразования. Это позволяет электрические цепи, контактирующие с инвертирующим и неинвертирующим входами ОУ, выполнить симметрично и реализовать свойство дифференциального входа ОУ подавления синфазных помех.

\section{4. Экспериментальные исследования макета нелинейного АЦП}

Разработанный макет был испытан в нормальных условиях с целью выявления параметров разброса выходных кодов в диапазоне изменения входного тока от 0 до 1000 нА. В качестве источника тока наноамперного диапазона использовался источник-измеритель Keithley 2450. Входной ток задавался в виде ступенчато изменяющегося тока, время экспозиции каждой ступени, в течение которого входной ток не менялся, составляло около 5 с. Выходные коды в виде текстового файла через USB разъем поступали в компьютер, в котором на экране монитора отображались в виде точек, показывающих изменения выходного кода $i$-го канала во времени. В каждом массиве кодов, соответствующем заданной ступени входного тока вычислялись среднее значение и стандартное отклонение. Разброс выходных кодов определяется наличием внешних помех, действующих на входные цепи преобразователя, среди которых всегда присутствует 
флуктуационный шум, и несовершенством работы электрической схемы, которое связано с шумами, генерируемыми элементами электрической схемы. Результаты преобразования токов в диапазоне от 0 до 1000 нА в код для одного из каналов приведены в таблице.

Цифровые данные, полученные на выходе одного канала преобразователя АЦ-ФОП при ступенчатом изменении входного измеряемого тока

\begin{tabular}{|c|c|c|c|c|c|}
\hline № & $\begin{array}{c}\text { Величина } \\
\text { входного } \\
\text { тока, } \\
\text { нА }\end{array}$ & $\begin{array}{c}\text { Среднее значение } \\
\text { цифрового } \\
\text { отсчета, М }\end{array}$ & $\begin{array}{c}\text { Стандартное } \\
\text { отклонение, } \\
\Delta\end{array}$ & $\begin{array}{c}\text { Относительное } \\
\text { значение стандартного } \\
\text { отклонения от среднего } \\
\text { значения, } \Delta / \mathrm{M}, \%\end{array}$ & $\begin{array}{c}\text { Время } \\
\text { экпоцици, } \\
\text { сек }\end{array}$ \\
\hline 1 & 0 & 1220,8 & 2,701 & 0,221 & 5,0 \\
\hline 2 & 1 & 1677,9 & 1,748 & 0,104 & 4,8 \\
\hline 3 & 2 & 2023,6 & 1,451 & 0,072 & 4,9 \\
\hline 4 & 4 & 2567,7 & 1,191 & 0,046 & 5,1 \\
\hline 5 & 8 & 3388,4 & 0,963 & 0,028 & 4,9 \\
\hline 6 & 16 & 4590,4 & 0,749 & 0,016 & 4,7 \\
\hline 7 & 32 & 6330,4 & 0,542 & 0,009 & 4,9 \\
\hline 8 & 64 & 8821,7 & 0,498 & 0,006 & 4,8 \\
\hline 9 & 128 & 12374,3 & 0,442 & 0,004 & 4,9 \\
\hline 10 & 256 & 17432,4 & 0,464 & 0,003 & 5,0 \\
\hline 11 & 512 & 24639,4 & 0,472 & 0,002 & 4,9 \\
\hline 12 & 1000 & 34559,8 & 0,384 & 0,001 & 5,0 \\
\hline
\end{tabular}

Из приведенных в таблице данных следует, что влияние флуктуационных шумов на стандартное отклонение от среднего значения при нелинейной шкале, аппроксимируемой степенной функцией с показателем степени $m$, близким к 0,5 , с увеличением входного тока уменьшается. При этом в области малых значений входного тока стандартное отклонение относительно среднего значения выходного кода не превышает $0,22 \%$, а на верхней границе динамического диапазона стандартное отклонение составляет $0,001 \%$ от среднего значения выходного кода. Разрешающая способность в начале шкалы не больше 10 пА.

Для оценки устойчивости работы электрической схемы производилось измерение стабильности выходных кодов преобразователя при ступенчатом изменении величины входного тока непрерывно в течение 14 ч работы преобразователя в нормальных условиях.

Результаты исследований функционирования макета аналого-цифрового преобразователя с нелинейной шкалой показали, что преобразование токов наноамперного диапазона в цифровые коды в нормальных условиях осуществляется с высокой стабильностью, которая характеризуется разбросом выходных кодов при неизменном входном сигнале во всем измеряемом наноамперном диапазоне не более $0,1 \%$ от величины выходного кода.

Поскольку исследуемый макет содержит 4 одинаковых канала преобразования, была произведена оценка межканальных помех для двух вариантов включения каналов:

1. На входы трех каналов подавался нулевой входной ток, а на вход 4-го канала меандр с входным током 0 нА и 1000 нА и периодом 900 мс.

2. На вход одного канала - нулевой входной ток, а на 3 других входа подавался один и тот же сигнал в виде меандра с входным том 0 нА и 1000 нА и периодом 900 мс.

В обоих вариантах обнаружить влияние меандра на выходные коды каналов с нулевым входным током не удалось. 


\section{5. Применение нелинейного измерителя малых токов для исследования магнитострикции}

Разработанный макет преобразователя «наноамперный ток-код» был применен для исследования конструкционных материалов, которые предварительно подвергались длительным циклическим механическим воздействиям на растяжение. В частности, проводилось измерение магнитострикции сталей, применяемых для изготовления трубопроводов, крупногабаритных корпусов, в которых относительное изменение размеров из-за влияния магнитострикции составляет $10^{-6}[9]$.

В процессе измерений осуществляли запись выходных кодов одного канала макета 4-канального преобразователя «ток-код», подключенного через согласующий каскад, имеющий дифференциальный вход и недифференциальный выход, к тензорезистивному мосту, а на второй канал макета подавался сигнал от потенциалметра.

Выходные коды макета АЦП регистрировали в виде последовательности кодов во времени с дискретом 10 мс, а изменение кодов во времени непосредственно использовано для оценки относительного изменения продольных и поперечных размеров образцов при изменении магнитного поля.

Результаты измерения магнитострикции с использованием преобразователя «наноамперный ток-код» показывают:

- разработанный и описанный в данной работе АЦП с нелинейной шкалой преобразования позволяет получить малые разбросы измерений - средние относительные разбросы значений цифровых кодов за все время составили не более $7 \%$;

- чувствительность АЦП имеет порядок $10^{-10} \mathrm{~A}$, что обеспечивает измерение изменения сопротивления тензомоста 400 Ом с разрешающей способностью порядка $10^{-4}$ Ом.

\section{6. Выводы}

1. Разработанный макет 4-канального преобразователя «наноамперный ток-код» позволяет регистрировать наблюдаемые параметры в динамическом диапазоне не менее 60 дБ с малыми разбросами выходных кодов в реальном времени.

2. Высокие характеристики преобразования достигнуты применением нелинейной шкалы преобразования, модель которой представляет степенную функцию с показателем степени, меньше единицы, и примененной схемой включения входов интегрирующего операционного усилителя, позволяющей использовать его свойство подавления синфазных сигналов.

3. Выбранная модель нелинейной шкалы позволяет уменьшить влияние флуктуационых шумов и нестабильной работы элементов электрической схемы преобразователя на разброс его выходных кодов.

\section{Литература}

1. Aaltonen L. Integrated Interface Electronics for Capacitive MEMS Inertial Sensors : Doct. Diss. - Aalto Univ., Sc. Sci. Tech., Fac. Electr., Comm. Autom., Dep. Micro Nanosci. - 2010. $152 \mathrm{p}$.

2. A High-Performance Tunneling Accelerometer / E. Boyden, O. El. Rifai, B. Hubert, M. Karpman, D. Roberts // Term Project 6.777, Introduction to Microelectromechanical Systems / MIT Media Lab. - Spring, 1999. - 58 p.

3. Temperature-Compensated Nano-Ampere Current Reference Circuit with Subthreshold Metal-Oxide-Semiconductor Field-Effect Transistor Resistor Ladder / Yuji Osaki, Tetsuya Hirose, Nobutaka Kuroki, and Masahiro Numa // Jpn. J. Appl. Phys. - Vol. 50, no. 4S. - P. 04DE08-1-6. DOI: 10.1143/JJAP.50.04DE08. 
4. Current Mode Techniques for Sub-pico-Ampere Circuit Design / B. Linares-Barranco, T. Serrano-Gotarredona, R. Serrano-Gotarredona, C. Serrano-Gotarredona // Analog Integrated Circuits and Signal Processing. - 2004. - Vol. 38. - P. 103-119.

5. Digital processors limited by power; what's the upside? - URL: https://phys.org/news/ 2012-08-digital-processors-limited-power-upside.html

6. Гельман М. М. Системные аналого-цифровые преобразователи и процессоры сигналов. - М. : Мир, 1999. - 559 с.

7. Лукин Н. А., Рубин Л. С. Применение нелинейного функционального аналогоцифрового преобразования для прецизионных измерений малых электрических величин в реальном времени // Гироскопия и навигация. - 2014. - № 4 (87) - С. 131-141.

8. Аналого-цифровой преобразователь интегрирующего типа для измерения малых электрических сигналов : пат. 2693647 C1 Рос. Федерация / Лукин Н.А., Рубин Л. С. № 20181056452019 ; заявл. 14.02.2018 ; опубл. 03.07.2019, Бюл. 19.

9. Some features in the behavior of magnetic and acoustic characteristics of hot-rolled 08G2B steel under cyclic loading / E. S. Gorkunov, A. M. Povolotskaya, S. M. Zadvorkin, E. A. Putilova, A. N. Mushnikov, E. G. Bazulin, A. K. Vopilkin // Russian Journal of Nondestructive Testing. 2019. - Vol. 55, no. 11. - P. 827-836. 